REVISTA INTERNACIONAL DE CIENCIAS DEL DEPORTE International Journal of Sport Science

Rev. int. cienc. deporte

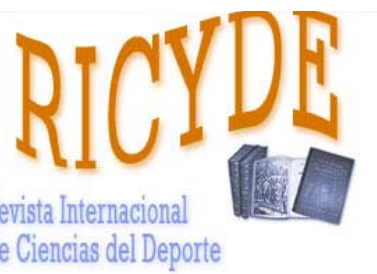

International Journal of Sport Science VOLUMEN VII - AÑO VII

Páginas:14-23 ISSN:1885-3137

No 22 - Enero - 2011

\title{
Diferencias en la distancia de lanzamiento y velocidad de balón según el puesto específico en jugadores de balonmano sub-18 \\ Differences in the throwing distance and ball velocity by playing position in under-18 handball players
}

\author{
Jesús Rivilla-García \\ Universidad Politécnica Madrid \\ Isidoro Martínez Martín \\ Universidad de León \\ Fernando Navarro Valdivielso \\ Universidad de Castilla La Mancha \\ Javier Sampedro Molinuelo \\ Universidad Politécnica Madrid
}

\section{Resumen}

El presente estudio ha analizado las diferencias entre puestos específicos ofensivos en la distancia de lanzamiento con balón medicinal pesado y liviano y en la velocidad de lanzamiento con y sin oposición en jugadores en formación. Para ello, cincuenta y ocho jugadores realizaron pruebas de progresiva especificidad: lanzamiento con balón medicinal pesado (LBMP) y ligero (LBML), velocidad de lanzamiento sin (VL) y con oposición (VLO).

VLO fue menor a VL en todos los puestos específicos, con diferencias significativas en los jugadores laterales $(p<0,01)$ y pivotes $(p<0,05)$, constatándose una influencia negativa de la oposición en la velocidad de lanzamiento. Igualmente, se constataron diferencias significativas $(p<0,001)$ entre puestos específicos en LBMP $(F 4,53=17,012)$, $\operatorname{LBML}(F 4,53=37,433)$, VL $(F 4,53=25,183)$ y VLO $(F 4,53=17,091)$, lo cual ratifica que el puesto específico podría ser determinante en la distancia de lanzamiento con balón medicinal y en la velocidad de lanzamiento en jugadores de balonmano en etapas formativas.

Palabras clave: lanzamiento de balón medicinal; velocidad de lanzamiento; adolescentes; puesto específico ofensivo; oposición.

\begin{abstract}
This study aims to analyze the differences in throwing distance with overweight ball and throwing velocity without and with opposition according to the playing positions in trainees handball players. For this purpose, fifty-eight players were assessed in four specific progress throwing situations: throwing with heavy medicinal ball (THMB) and light medicinal ball (TLMB), throwing velocity without (TV) and with opposition (TVO).

TVO was less than TV in all playing positions, with significant differences in back $(p<0.01)$ and pivot players $(p<0.05$ ), confirming a negative influence of the opposition in the throwing velocity.

Significant differences $(p<0.001)$ were found among the playing positions in THMB $(F 4,53=17.012)$, TLMB $(F 4,53=37.433)$, TV (F4,53=25.183) and TVO ( F4, 53=17.091), which confirms that playing position could be decisive in the throwing distance and throwing velocity in handball players of formative stages.
\end{abstract}

Key words: medicine ball throw; throwing velocity; opposition, adolescents; playing position.

Correspondencia/correspondence: Jesús Rivilla García

C/. Martín Fierro, 7. Facultad de Ciencias de la Actividad Física y del Deporte Universidad Politécnica de Madrid. 28040 Madrid

e-mail: jesus.rivilla@upm.es 
Rivilla, J.; Martínez, M.; Navarro, F.; Sampedro, J. (2011). Diferencias en la distancia de lanzamiento y velocidad de balón según el puesto específico en jugadores de balonmano sub-18. Revista Internacional de Ciencias del Deporte, 21(6), 14-23. http://www.cafyd.com/REVISTA/02202.pdf

\section{Introducción}

$E^{1}$ lanzamiento y su eficacia son claves para ganar partidos (Carreras, 1992), siendo la velocidad del balón y la precisión determinantes en la consecución del gol (Fleck et al., 1992; Van den Tillaar, y Ettema, 2003). Quizá por ello, numerosos estudios han evaluado esta acción del juego, tanto con pruebas de lanzamiento con balón medicinal (Lidor et al., 2005; Rogulj, Srhoj, Nazor, Srhoj, y Cavala, 2005; Visnapuu, y Jurimae, 2008) como con test de velocidad de lanzamiento (Bayios, Anastasopoulou, Sioudris, y Boudolos, 2001; Bretagne, 1980; Cardoso, y González-Badillo, 2006; Fleck et al., 1992; Joris, Edwards, Van Ingen Schenau, y Kemper, 1985; Marques, Van den Tillaar, Vescovi, y González-Badillo, 2007; Mikkelsen, y Olensen, 1985; Prokajac, 1980; Šibila, Pori, y Bon, 2003).

Los jugadores pueden ser clasificados según su puesto específico ofensivo: laterales, centrales, pivotes, extremos y porteros (Marczinka, 1993; Pezarat-Correia, Valamatos, Alves, y Santos, 2007; Šibila, Vuleta, y Pori, 2004). Los entrenadores de alto nivel han afirmado que la fuerza de lanzamiento es más importante para los laterales y centrales que para los extremos y pivotes, y poco relevante para los porteros (Moreno, 2004).

Pese a que se han constatado diferencias en la condición física general y velocidad de lanzamiento según el grado competitivo y edad (Gorostiaga, Granados, Ibanez, y Izquierdo, 2005; Granados, Izquierdo, Ibanez, Bonnabau, y Gorostiaga, 2007; Vila, Fernández, y Rodríguez, 2007), son escasos los estudios sobre el lanzamiento en función del puesto específico, habiéndose realizado con muestras del sexo femenino. Así, en jugadoras en formación, las laterales lanzaron a mayor velocidad que el resto, hallando diferencias significativas con el portero (Zapartidis et al., 2009). Por el contrario, en jugadoras de élite junior y sénior no se encontraron diferencias en el lanzamiento con balón medicinal, si bien los porteros consiguieron los peores valores (Rogulj et al., 2005).

En las acciones y distancias recorridas en el juego se han constatado diferencias entre puestos (Cambel, 1985; Luig et al., 2008; Manchado, Hoffmann, Navarro, y Platen, 2007; Ziv, y Lidor, 2009; Šibila et al., 2004), confirmándose diferencias en el número de lanzamientos (Madrera, Herrero, Fernández, y Martínez, 2003). Asimismo, se han hallado diferencias en las características antropométricas (Cavala, Rogulj, Srhoj, y Katic, 2005; Chaouachi et al., 2009; Rogulj et al., 2005; Ruiz, Rodríguez, y Enrique, 2001; Srhoj, Marinovic, y Rogulj, 2002; Taborsky, 2007), condición física y habilidades motoras (Oxyzoglou, Hatzimanouil, Kanioglou, y Papadopoulou, 2008; Pezarat-Correia et al., 2007; Vasques, Antunes, Silva, y Lopes, 2005; Zapartidis et al., 2009) y caracteristicas psicológicas (Rogulj et al., 2005), no siendo así en determinadas capacidades fisiológicas (Chaouachi et al., 2009; Manchado et al., 2007).

A la luz de las escasas aportaciones referidas al análisis del lanzamiento en función del puesto específico, y teniendo en cuenta las importantes diferencias, el presente estudio se realizó con el propósito de comprobar las diferencias entre los distintos puestos específicos ofensivos en las pruebas de lanzamiento con balón medicinal pesado y ligero, y la velocidad de lanzamiento con y sin oposición en jugadores de balonmano en formación. Se consideró importante incluir tanto pruebas de menor especificad, como los lanzamientos con balón medicinal, como test específicos de velocidad de lanzamiento. En este sentido, nuestro estudio incluyó un test de velocidad de lanzamiento con oposición, teniendo en cuenta que la velocidad del balón no sólo depende de factores físico-técnicos (Joris et al., 1985; Van Muijen, Joris, Kemper, y Van Ingen Schenau, 1991) y del grado de precisión (Bayios, y Boudolos, 1998; Van den Tillaar, y Ettema, 2003), sino también de factores cognitivos y de oposición (Fleck et al., 1992), como así lo corroboran algunos estudios en balonmano (López, 
Rivilla, J.; Martínez, M.; Navarro, F.; Sampedro, J. (2011). Diferencias en la distancia de lanzamiento y velocidad de balón según el puesto específico en jugadores de balonmano sub-18. Revista Internacional de Ciencias del Deporte, 21(6), 14-23. http://www.cafyd.com/REVISTA/02202.pdf

2005; Pardo, González, y Mayo, 2007; Párraga et al., 2001; Párraga, Sánchez, y Oña, 2001; Rivilla, Sampedro, Navarro, y Gómez, 2010) y waterpolo (Van der Wende, 2005; Vila et al., 2009).

La hipótesis de partida fue que existen diferencias significativas en los test generales y específicos del lanzamiento en función de los puestos específicos, obteniendo mejores valores los puestos específicos de $1^{\mathrm{a}}$ línea (lateral y central) tras los de $2^{\mathrm{a}}$ línea (pivote y extremo) y, por último, los porteros. Su confirmación supondría importantes consecuencias para el entrenamiento y su adecuación a las necesidades de cada puesto específico, asimismo, la evaluación de esta acción del juego, desde el punto de vista condicional, debería adecuarse en gran medida al puesto específico del jugador evaluado, siendo los valores de estos test relevantes para la selección y asignación del puesto específico.

\section{Método}

\section{Participantes}

La muestra estuvo formada por 58 jugadores de balonmano, con edades comprendidas entre 15 y 18 años, pertenecientes a cuatro equipos de Ciudad Real de diferentes categorías federadas: cadete y juvenil. La muestra se dividió de acuerdo al puesto específico (tabla 1): lateral $(\mathrm{L})$, central $(\mathrm{C})$, pivote $(\mathrm{P})$, extremo $(\mathrm{E})$ y portero $(\mathrm{G})$.

Tabla 1. Características generales de la muestra $(\mathrm{X} \pm \mathrm{DT})$

\begin{tabular}{|l|c|c|c|c|c|}
\hline PUESTO ESPECÍFICO & $N$ & EDAD (a) & ESTATURA $(\mathrm{cm})$ & PESO $(\mathrm{kg})$ & OBSERVACIONES \\
\hline Laterales (L) & 15 & $17,3 \pm 1,03$ & $178 \pm 3,19$ & $75,4 \pm 5,76$ & $\begin{array}{c}\text { Juvenil }(\mathrm{n}=11) \\
\text { Cadete }(\mathrm{n}=4)\end{array}$ \\
\hline Centrales (C) & 10 & $17,02 \pm 1,83$ & $172 \pm 5,97$ & $71,25 \pm 8,9$ & $\begin{array}{c}\text { Juvenil }(\mathrm{n}=8) \\
\text { Cadete }(\mathrm{n}=2)\end{array}$ \\
\hline Pivotes (P) & 13 & $16,51 \pm 1,65$ & $173 \pm 4,46$ & $77,48 \pm 7,3$ & $\begin{array}{c}\text { Juvenil }(\mathrm{n}=11) \\
\text { Cadete }(n=2)\end{array}$ \\
\hline Extremos (E) & 13 & $16,89 \pm 2,08$ & $170 \pm 7,24$ & $72,3 \pm 8,8$ & $\begin{array}{c}\text { Juvenil }(n=11) \\
\text { Cadete }(n=2)\end{array}$ \\
\hline Porteros (G) & 7 & $17,15 \pm 2,21$ & $177 \pm 5,59$ & $73,32 \pm 3,6$ & $\begin{array}{c}\text { Juvenil }(n=5) \\
\text { Cadete }(n=2)\end{array}$ \\
\hline
\end{tabular}

\section{Procedimiento}

Los participantes fueron valorados en cuatro situaciones de creciente especificidad: 1) lanzamiento con balón medicinal pesado (LBMP), 2) lanzamiento con balón medicinal ligero (LBML), 3) velocidad de lanzamiento sin oposición (VL) y 4) velocidad de lanzamiento con oposición (VLO). Con el fin de reducir las interferencias del entorno y condicionantes externos (calendario, carga de entrenamientos anteriores, exigencia de competición anterior, lesiones, asistencia,...), cada equipo fue evaluado en una sola sesión, elegida por los investigadores y entrenadores de cada equipo con los criterios principales de búsqueda de estado óptimo de recuperación y asistencia de la mayoría de los componentes del equipo. Posteriormente, debido a la selección de una sola sesión de evaluación, se optó porque todos los equipos ejecutaran las pruebas en el mismo orden, de menor a mayor especificidad. 
Rivilla, J.; Martínez, M.; Navarro, F.; Sampedro, J. (2011). Diferencias en la distancia de lanzamiento y velocidad de balón según el puesto específico en jugadores de balonmano sub-18. Revista Internacional de Ciencias del Deporte, 21(6), 14-23. http://www.cafyd.com/REVISTA/02202.pdf

\section{Lanzamientos con balón medicinal pesado y ligero}

En ambos lanzamientos el propósito era lanzar el móvil a la mayor distancia posible, no pudiéndose mover el lanzador más allá de la línea de lanzamiento. El test con balón de $3 \mathrm{~kg}$ consistió en ejecutar un lanzamiento, con ambas manos por encima y detrás de la cabeza, mediante un movimiento explosivo hacia delante (Martínez, 2002). Mientras que el lanzamiento con balón de $0,8 \mathrm{~kg}$ consistió en lanzar el balón, adaptado con una mano, efectuando tres pasos previos y finalizando con el pie contrario al brazo ejecutor, con similar técnica a la de un pase largo en balonmano (Torrescusa, 1986).

\section{Velocidad de lanzamiento sin y con oposición}

Las indicaciones comunes a ambas pruebas fueron: lanzar en apoyo a la mayor velocidad posible, utilizando una sola mano y procedimiento propio de lanzamiento a portería, con un máximo de tres pasos previos y por detrás de la línea de 9 metros.

Con el objetivo de simular la acción real de juego, se permitió el uso de resina en las manos. Los lanzamientos debían ser precisos conforme al criterio descrito por Zeier (1987): en la prueba de VL (sin portero) los lanzamientos debían ser encaminados a las esquinas de la portería mientras que en VLO (con portero) debían dirigirse a las esquinas, más alejadas del portero. Éste se ubicó en una línea a $0,5 \mathrm{~m}$ de la portería permitiéndosele realizar movimientos exclusivamente en el plano frontal, un observador registró su actuación mediante visionado a cámara lenta de cada secuencia de lanzamiento desde un plano lateral al portero. Los entrenadores comprobaron la ejecución para certificar el uso de la técnica correcta del lanzamiento en apoyo con armado clásico, los cuales habían sido entrenados previamente para la correcta percepción y análisis de las acciones relevantes del juego hasta lograr un óptimo nivel en dichas capacidades (Rivilla-García, Díaz y Sampedro, 2010).

Cada participante, en cada una de las pruebas, ejecutó lanzamientos hasta quedar registrados tres valores en cada una de las pruebas, quedando verificados los dos mejores valores de cada prueba, escogiéndose el mejor valor de cada prueba para el posterior análisis.

El proceso fue el siguiente: cada sujeto realizaba una serie de tres lanzamientos continuados con una pausa entre ellos de 10-15 segundos; si era preciso efectuar más lanzamientos por no haber obtenido los tres registros, el jugador realizaba una segunda serie de lanzamientos con un descanso entre series de 1-2 minutos, estableciéndose un máximo de tres series de lanzamiento (Gorostiaga et al., 2005; Granados et al., 2007). Igualmente, se estableció una pausa mínima de 5 minutos en el paso de una prueba a otra para garantizar la recuperación total del jugador. Ningún jugador realizó más de 15 lanzamientos en total, evitando así la fatiga por acumulación de acciones explosivas.

Con objeto de motivar a los jugadores se les informó sobre la distancia y velocidad del lanzamiento seguidamente después de la ejecución de cada intento.

El coeficiente de correlación intraclase (ICC) fue de 0,98 (LBMP), 0,97 (LBML), 0,98 (VL) y 0,97 (VLO) y el coeficiente de variación (CV) fue de 4,1\% (LBMP), 7,4\% (LBML), 4,4\% (VL) y $5,1 \%$ (VLO).

\section{Material}

Las pruebas se efectuaron en un pabellón cerrado de balonmano. Se utilizaron dos balones marca "Salter" de 3 y $0,8 \mathrm{~kg}$. con una circunferencia de 72,22 y $58 \mathrm{~cm}$. para las pruebas con balón medicinal. La distancia se midió con cinta métrica de resolución $0,01 \mathrm{~m}$. La precisión exacta del lugar de caída del balón medicinal se realizó mediante una lona (20 x 3 m) color negro en la cual se marcaba el balón al contacto con la misma. 
Rivilla, J.; Martínez, M.; Navarro, F.; Sampedro, J. (2011). Diferencias en la distancia de lanzamiento y velocidad de balón según el puesto específico en jugadores de balonmano sub-18. Revista Internacional de Ciencias del Deporte, 21(6), 14-23. http://www.cafyd.com/REVISTA/02202.pdf

Para las pruebas de velocidad de lanzamiento, se utilizaron balones reglamentarios oficiales, de 480 gr de peso y $58 \mathrm{~cm}$ de circunferencia. La velocidad de lanzamiento fue evaluada a partir del tiempo transcurrido desde que el balón franqueaba la línea de $6 \mathrm{~m}$ hasta que contactaba con la portería, situada a $6 \mathrm{~m}$ de la portería, o con el portero, situado a 5,5 $\mathrm{m}$ de dicha línea. Por tanto, el cálculo de la velocidad de lanzamiento se realizó mediante el tiempo vuelo del balón hasta el impacto con portero o portería y la distancia de $6 \mathrm{~m}$ (portería) o 5,5 $\mathrm{m}$ (portero). El registro del tiempo fue realizado con una precisión de 0,001 s, utilizando un sistema de cronometraje (Sportmetrics, Valencia, España) compuesto por un sensor de paso integrado por células fotoeléctricas y un sensor de sonido. El sensor de paso, ubicado en la línea de $6 \mathrm{~m}$, con una separación de $2 \mathrm{~m}$, estaba compuesto por ocho células fotoeléctricas distribuidas vertical y uniformemente, con una separación entre ellas de $15 \mathrm{~cm}$ (rango de 1,40-2,50 m sobre el suelo). El sensor de sonido, de intensidad regulable, se instaló en la parte inferior central de la portería. La portería estaba compuesta por una superficie de metal que producía un sonido detectable por el sensor de sonido al contacto con el balón. El cronometraje del tiempo comenzaba automáticamente cuando el balón pasaba por las fotocélulas y era detenido cuando el balón contactaba con la portería o con el portero. Ya que la distancia a la que impactó el balón hasta el sensor del sonido no superó en ningún caso a una distancia superior a $2,5 \mathrm{~m}$, y puesto que el sonido genera un retraso de $0,001 \mathrm{~s}$ cada 30 $\mathrm{cm}$., se estima la existencia de un error de medida no superior a 0,008 s. Los movimientos del portero en el plano frontal (VLO) fueron controlados con una cámara situada en la prolongación lateral del mismo.

\section{Análisis estadístico}

Se calcularon las medias y las desviaciones típicas de las variables LBMP, LBML, VL y VLO. El análisis de diferencias entre puestos específicos en las variables dependientes se efectuó mediante la prueba Anova de un Factor, aplicándose un análisis post hoc mediante el método de Bonferroni. Para analizar las diferencias entre la VL y la VLO en los puestos se aplicó la prueba $\mathrm{T}$ de Student para muestras relacionadas. Los cálculos estadísticos se llevaron a cabo con el programa estadístico SPSS 10.0.

\section{Resultados}

Los resultados revelaron significativas diferencias entre los puestos específicos en los cuatro test de lanzamiento (tabla 2), LBMP $\left(\mathrm{F}_{4},{ }_{53}=17,012 ; \mathrm{p}<0,001\right)$, LBML $\left(\mathrm{F}_{4}, 53=37,433\right.$; $\mathrm{p}<0,001), \operatorname{VL}\left(\mathrm{F}_{4,53}=25,183 ; \mathrm{p}<0,001\right)$ y $\operatorname{VLO}\left(\mathrm{F}_{4,53}=17,091 ; \mathrm{p}<0,001\right)$.

Tabla 2. Valores $(\mathrm{X} \pm \mathrm{DT})$ obtenidos en las pruebas de lanzamiento por los puestos específicos.

\begin{tabular}{|l|c|c|c|c|c|}
\hline $\begin{array}{c}\text { Tests de } \\
\text { lanzamiento }\end{array}$ & $\begin{array}{c}\text { Laterales } \\
(\mathrm{n}=15)\end{array}$ & $\begin{array}{c}\text { Centrales } \\
(\mathrm{n}=10)\end{array}$ & $\begin{array}{c}\text { Pivotes } \\
(\mathrm{n}=13)\end{array}$ & $\begin{array}{c}\text { Extremos } \\
(\mathrm{n}=13)\end{array}$ & $\begin{array}{c}\text { Porteros } \\
(\mathrm{n}=7)\end{array}$ \\
\hline LBMP $(\mathrm{m})$ & $10,98 \pm 1,15^{\mathrm{cpeg}}$ & $9,72 \pm 0,74^{\mathrm{g}}$ & $9,71 \pm 1,15^{\mathrm{lg}}$ & $8,85 \pm 1,73^{\mathrm{l}}$ & $7,74 \pm 1,31^{\mathrm{lcp}}$ \\
\hline LBML $(\mathrm{m})$ & $36,14 \pm 3,79^{\text {peg }}$ & $31,98 \pm 5,19^{\text {peg }}$ & $25,48 \pm 3,51^{\mathrm{cg}}$ & $23,59 \pm 2,02^{\mathrm{lc}}$ & $20,63 \pm 1,69^{\mathrm{cp}}$ \\
\hline VL $\left(\mathrm{m} \cdot \mathrm{s}^{-1}\right)$ & $24,17 \pm 1,29^{\text {peg }}$ & $22,85 \pm 0,91^{\text {peg }}$ & $20,87 \pm 1,34^{\mathrm{lc}}$ & $20,30 \pm 1,12^{\mathrm{cc}}$ & $19,84 \pm 1,72^{\mathrm{lc}}$ \\
\hline VLO $\left(\mathrm{m}^{-1} \mathrm{~s}^{-1}\right)$ & $22,33 \pm 1,74^{\text {peg }}$ & $22,13 \pm 1,52^{\text {peg }}$ & $19,43 \pm 1,21^{\mathrm{lc}}$ & $19,35 \pm 1,17^{\mathrm{cc}}$ & $18,42 \pm 1,28^{\mathrm{lc}}$ \\
\hline
\end{tabular}

Significativas diferencias $(p<0,05)$ : 'Diferencia significativa con laterales. 'Diferencia significativa con centrales. PDiferencia significativa con pivotes. ${ }^{\mathbf{E}}$ Diferencia significativa con extremos. gSignificativa diferencia con porteros. 
Rivilla, J.; Martínez, M.; Navarro, F.; Sampedro, J. (2011). Diferencias en la distancia de lanzamiento y velocidad de balón según el puesto específico en jugadores de balonmano sub-18. Revista Internacional de Ciencias del Deporte, 21(6), 14-23. http://www.cafyd.com/REVISTA/02202.pdf

Se observó una tendencia similar de resultados en los cuatro lanzamientos (figura 1) en los cuales los laterales obtuvieron los valores más altos, seguidos de los centrales, tras ellos los pivotes y extremos, con escasas diferencias entre ellos en VL y VLO, y en último lugar los porteros.

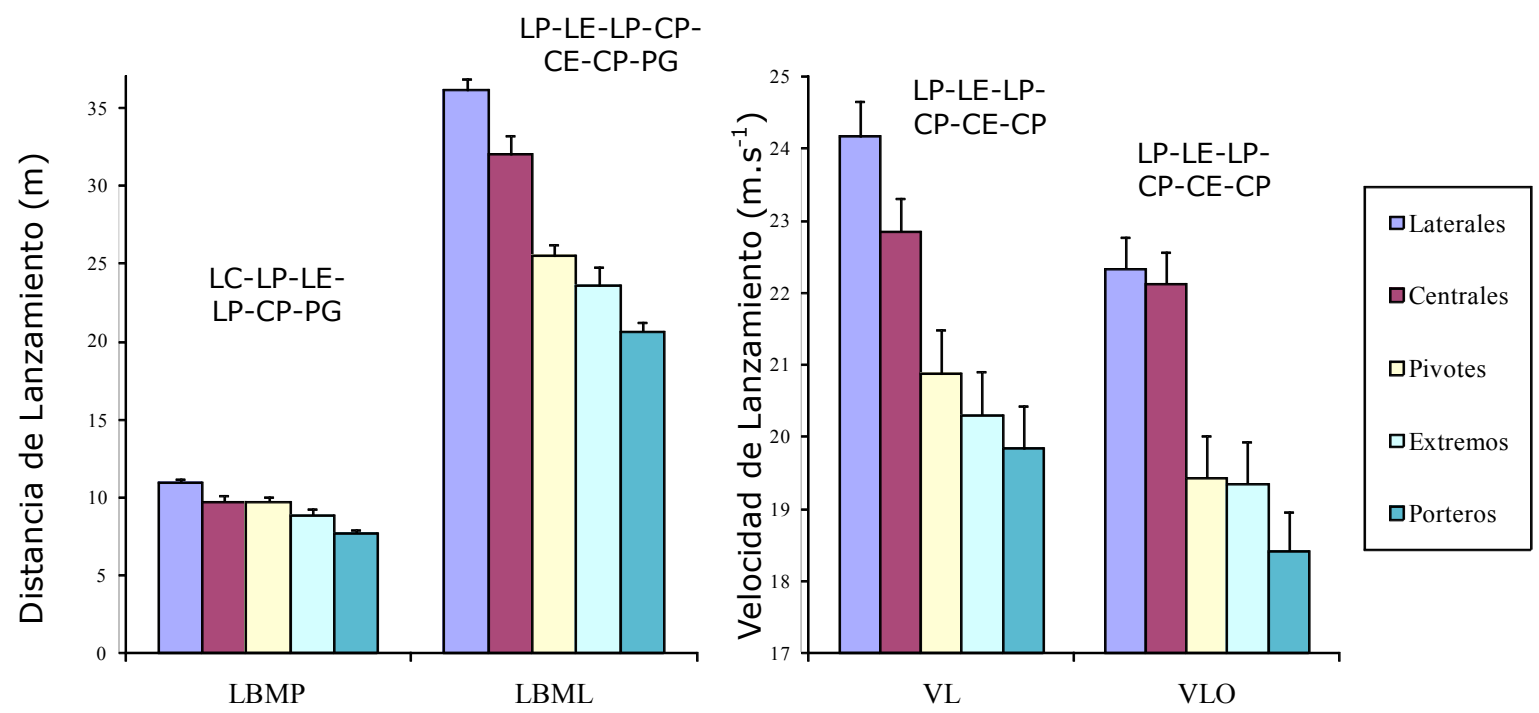

Figura 1. Valores $(\mathrm{X} \pm \mathrm{DT})$ obtenidos en las pruebas de lanzamiento en función del puesto específico. Significativas diferencias $(p<0,05)$ entre: ${ }^{\mathrm{LC}}$ Lateral-Central, ${ }^{\mathrm{LP}}$ Lateral-Pivote, ${ }^{\mathrm{LE}}$ Lateral-Extremo, ${ }^{\mathrm{LG}}$ LateralPortero, ${ }^{\mathrm{CP}}$ Central-Pivote, ${ }^{\mathrm{CE}}$ Central-Extremo, ${ }^{\mathrm{CG}}$ Central-Portero, ${ }^{\mathrm{PE}}$ Pivote-Extremo, ${ }^{\mathrm{PG}}$ Pivote-Portero, ${ }^{\mathrm{EG}}$ Extremo-Portero

Los laterales fueron significativamente superiores a los porteros y extremos en los cuatro test $(p<0,001)$, a los pivotes en LBMP $(p=0,003)$, en LBML $(p<0,001)$, en VL $(p<0,001)$ y en VLO $(p<0,001)$, y a los centrales en LBMP $(p=0,019)$. Los centrales obtuvieron valores significativamente superiores a los porteros en los cuatro test $(p<0,001)$, a los extremos en LBML $(p<0,001)$, en VL $(p<0,001)$ y en VLO $(p<0,001)$. Los pivotes fueron significativamente mejores que los porteros en LBMP $(p<0,001)$ y en LBML $(p=0,049)$. No hubo diferencias significativas entre los extremos y pivotes en los cuatro test, ni tampoco entre los laterales y centrales en las tres pruebas más específicas.

Por otra parte, la VL fue superior a la VLO en todos los puestos específicos (figura 2). 
Rivilla, J.; Martínez, M.; Navarro, F.; Sampedro, J. (2011). Diferencias en la distancia de lanzamiento y velocidad de balón según el puesto específico en jugadores de balonmano sub-18. Revista Internacional de Ciencias del Deporte, 21(6), 14-23. http://www.cafyd.com/REVISTA/02202.pdf

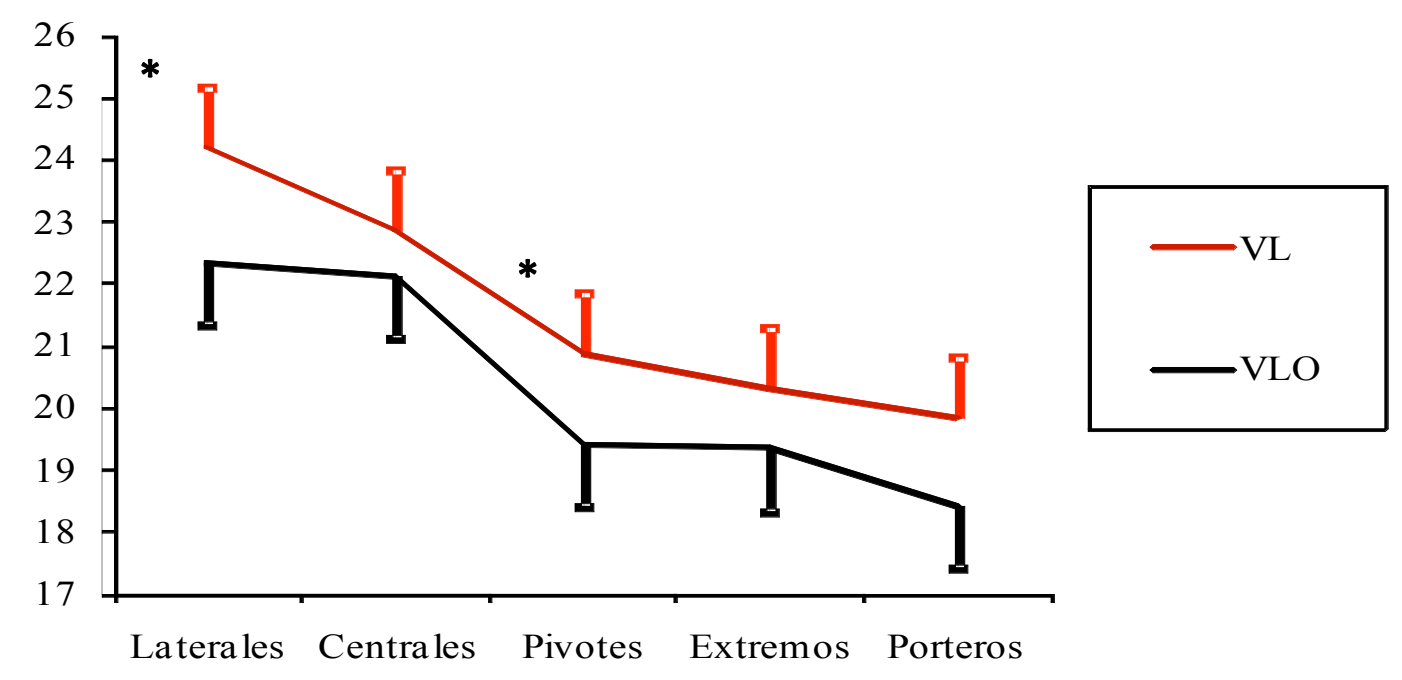

Figura 2. Valores $(\mathrm{X} \pm \mathrm{DT})$ de velocidad de lanzamiento sin y con oposición en los diferentes puestos específicos ofensivos. *Diferencias significativas $(p<0,05) * *$ Diferencias significativas $(p<0,01)$.

Se constataron diferencias significativas en el puesto específico de lateral (TV$\left.\mathrm{TVO}=1,842 \pm 1,01 \mathrm{~m} \cdot \mathrm{s}^{-1} ; \mathrm{p}<0,001 ; \mathrm{t}=7,077 ; \mathrm{gl}=14\right)$ y de pivote $\left(\mathrm{TV}-\mathrm{TVO}=1,431 \pm 1,5 \mathrm{~m} \cdot \mathrm{s}^{-1}\right.$; $\mathrm{p}=0,005 ; \mathrm{t}=3,435 ; \mathrm{gl}=12$ ), no siendo significativas en el portero, extremo y central.

\section{Discusión}

A través de la revisión bibliográfica se constató la existencia de escasas investigaciones que analizaran las diferencias en la distancia y velocidad del lanzamiento entre los diferentes puestos específicos ofensivos. Sorprende el hecho de que, aún siendo muy utilizado en el ámbito del entrenamiento deportivo (Van Muijen et al., 1991) así como en la evaluación de la condición física en balonmano (Mayo y Pardo, 2001; Moreno, 2004), apenas se encontraron estudios en balonmano que usaran el lanzamiento con balón medicinal pesado o ligero (Rogulj et al., 2005), no así en otros deportes como el béisbol donde el lanzamiento es relevante (DeRenne, Ho y Blitzblau, 1990; Brylinsky, Moore y Frosch, 1992; Newton y McEvoy, 1994; Escamilla et al., 2000). Igualmente, no se encontraron estudios comparativos que incluyeran pruebas con algún tipo de oposición.

Acerca de los resultados obtenidos, el orden de los valores obtenidos en cada puesto específico fue similar en los cuatro test: el lateral fue el mejor, seguido de los centrales, pivotes, extremos y, en último lugar, los porteros. Las diferencias significativas, en las cuatro pruebas, fueron encontradas fundamentalmente entre los puestos que obtuvieron mejores valores (lateral y central) y los que obtuvieron peores valores (portero, extremos y pivotes). Ello podría significar que el puesto específico ofensivo es determinante en el lanzamiento en balonmano en jugadores en formación. Las razones de estas diferencias, podrían deberse tanto a la preparación específica, ya que los puestos de la primera línea realizan un mayor número de lanzamientos a distancia en entrenamientos y partidos, como a la asignación de puestos realizada por los entrenadores en el proceso de especialización. En cualquier caso, los resultados constatan la necesidad de adecuar el entrenamiento condicional del lanzamiento a las necesidades y características de cada puesto específico. Igualmente, las diferencias entre puestos específicos conforman una información relevante para la selección de jugadores en cada puesto específico de acuerdo a las medidas obtenidas por los jugadores en estos test. 
Rivilla, J.; Martínez, M.; Navarro, F.; Sampedro, J. (2011). Diferencias en la distancia de lanzamiento y velocidad de balón según el puesto específico en jugadores de balonmano sub-18. Revista Internacional de Ciencias del Deporte, 21(6), 14-23. http://www.cafyd.com/REVISTA/02202.pdf

Estos datos se encuentran en sintonía con los obtenidos por jugadoras de balonmano en edades de formación que mostraron diferencias significativas entre laterales y porteros en la velocidad de lanzamiento (Zapartidis et al., 2009), y en consonancia con el estudio de detección y selección de talentos donde los entrenadores de alto nivel afirmaron que la fuerza de lanzamiento era más importante para la primera línea que para la segunda, siendo poco relevante para los porteros (Moreno, 2004). Sin embargo, los datos referidos al lanzamiento con balón medicinal pesado contradicen los hallados en jugadoras de élite junior y senior (Rogulj et al., 2005) que no obtuvieron diferencias significativas, si bien las diferencias portero-lateral $(p=0,06)$ y portero-extremo $(p=0,06)$ estuvieron al borde de ser estadísticamente significativas.

Por otro lado, se constató que la velocidad de lanzamiento fue menor con oposición en todos los puestos específicos analizados, con significación en el lateral y pivote. Puesto que ambos test de velocidad de lanzamiento se distinguen únicamente por la oposición, cabría pensar que estas diferencias se deben a la influencia de los factores cognitivos en la velocidad de lanzamiento. Los valores concuerdan con los estudios que analizaron la velocidad de lanzamiento sin y con oposición, tanto en balonmano (Pardo et al., 2007; Rivilla et al., 2009) como en waterpolo (Van der Wende, 2005; Vila et al., 2009). Igualmente, (Párraga et al., 2001) Párraga y colaboradores obtuvieron valores de velocidad de lanzamiento, en salto con toma de decisión, inferiores a otras investigaciones realizadas sobre el mismo tipo de lanzamiento (Bayios et al., 2001; Šibila et al., 2003), aunque ello podría deberse a que, al haberse realizado un análisis bidimensional (2D), se despreció la componente transversal de la velocidad. Por el contrario, en jugadores de División de Honor B no se encontraron diferencias significativas en la velocidad de lanzamiento en salto sin y con oposición (López, 2005).

No obstante, el hecho de que se haya seguido el mismo orden en la ejecución de las pruebas en todos los participantes constituye una limitación del estudio, ya que podría conllevar un descenso de valores conforme aumenta la fatiga de los jugadores. Para evitarlo, se controló especialmente que el número de lanzamientos no fuera excesivo así como el descanso entre series. Igualmente, el hecho de mantener el orden en todos los participantes supondría un error progresivo que afectaría a todos los grupos o puestos específicos por igual. Por tanto, las posibles diferencias podrían ser atribuidas a las características del puesto específico. En cualquier caso, los resultados obtenidos deben ser valorados con especial cautela.

\section{Conclusiones}

Los resultados permiten confirmar la existencia de diferencias entre puestos específicos ofensivos tanto en la capacidad general como específica de lanzamiento, ratificándose una clasificación en función del puesto: lateral, central, pivote, extremo y portero. Por tanto, el puesto específico ofensivo determina la capacidad de lanzamiento en jugadores de etapas formativas. Consecuentemente, los datos del lanzamiento podrían ser relevantes en el proceso de especialización en el puesto de los jugadores en formación.

Por otro lado, la oposición influye de manera significativa en la velocidad de lanzamiento a portería, disminuyendo significativamente la misma en laterales y pivotes, y constatándose con ello la importancia de entrenar y valorar condicionalmente el lanzamiento en situaciones de oposición. 
Rivilla, J.; Martínez, M.; Navarro, F.; Sampedro, J. (2011). Diferencias en la distancia de lanzamiento y velocidad de balón según el puesto específico en jugadores de balonmano sub-18. Revista Internacional de Ciencias del Deporte, 21(6), 14-23. http://www.cafyd.com/REVISTA/02202.pdf

\section{Referencias}

Bayios, I. A.; Anastasopoulou, E. M.; Sioudris, D. S., y Boudolos, K. D. (2001). Relationship between isokinetic strength of the internal and external shoulder rotators and ball velocity in team handball. Journal of Sports Medicine \& Physical Fitness, 41(2), 229-235.

Bayios, I. A., y Boudolos, K. (1998). Accuracy and throwing velocity in handball. Proceedings of the XVIth International Symposium on Biomechanics in Sports (Edited by HJ Riehle and MM Vieten), 55-58.

Bretagne, T. (1980). Lance missiles du sport. Equipe Magazine, 15(10), 4-7.

Brylinsky, J.; Moore, J. C.; y Frosch, M. (1992). The effect of using a weighted softball on pitching velocity, wrist strength and handgrip. Journal of Applied Sport Science Research, 6(3), 170-173.

Cambel, K. (1985). An assessment of the movement requirements of elite team handball athletes. Sports Medicine, 3, 23-30.

Cardoso, M. A., y González-Badillo, J. J. (2006). In-season resistance training and detraining in professional team handball players. Journal of Strength \& Conditioning Research, 20(3), 563-571.

Carreras, J. (1992). Propuesta de una metodología para el perfeccionamiento del lanzamiento en balonmano en la etapa de iniciación. Apunts: Educación Física y Deportes, (30), 38-45.

Cavala, M.; Rogulj, N.; Srhoj, V., y Katic, R. (2005). Position-related differences in morphological characteristics of croatian top-level female handball players. Proceedings: 4th International Scientific Conference on Kinesiology - Science and Profession-Challenge for the Future, Opatija, Croatia, 7-11 September 2005. In Faculty of Kinesiology, University of Zagreb. Croatia. 504-506.

Chaouachi, A.; Brughelli, M.; Levin, G.; Boudhina, N. B. B.; Cronin, J., y Chamari, K. (2009). Anthropometric, physiological and performance characteristics of elite teamhandball players. Journal of Sports Sciences, 27(2), 151-157.

DeRenne, C.; Ho, K., y Blitzblau, A. (1990). Effects of weighted implement training on throwing velocity. Journal of Applied Sport Science Research, 4(1), 16-19.

Escamilla, R. F.; Speer, K. P.; Fleisig, G. S.; Barrentine, S. W., y Andrews, J.R. (2000). Effects of throwing overweight and underweight baseballs on throwing velocity and accuracy. Sports Medicine, 29(4), 259-272.

Fleck, S. J.; Smith, S. L.; Craib, M. W.; Denaham, T.; Snow, R. E., y Mitchell, M. L. (1992). Upper extremity isokinetic torque and throwing velocity in team handball. Journal of Applied Sport Science Research, 6(2), 120-124.

Gorostiaga, E. M.; Granados, C.; Ibanez, J., y Izquierdo, M. (2005). Differences in physical fitness and throwing velocity among elite and amateur male handball players. International Journal of Sports Medicine, 26(3), 225-232.

Granados, C.; Izquierdo, M.; Ibanez, J.; Bonnabau, H., y Gorostiaga, E. M. (2007). Differences in physical fitness and throwing velocity among elite and amateur female handball players. International Journal of Sports Medicine, 28(10), 860-867.

Joris, H.; Edwards, V. M.; Van Ingen Schenau, G. J., y Kemper, H. C. G. (1985). Force, velocity and energy flow during the overarm throw in female handball players. Journal of Biomechanics, 18(6), 409-414. 
Rivilla, J.; Martínez, M.; Navarro, F.; Sampedro, J. (2011). Diferencias en la distancia de lanzamiento y velocidad de balón según el puesto específico en jugadores de balonmano sub-18. Revista Internacional de Ciencias del Deporte, 21(6), 14-23. http://www.cafyd.com/REVISTA/02202.pdf

Lidor, R.; Falk, B.; Arnon, M.; Cohen, Y.; Segal, G., y Lander, Y. (2005). Measurement of talent in team handball: The questionable use of motor and physical tests. The Journal of Strength and Conditioning Research, 19(2), 318-325.

López, P. (2005). Efecto de la oposición sobre los factores biomecánicos del lanzamiento en salto en balonmano. (Tesis Doctoral, Universidad de Jaén). Jaén: Universidad de Jaén.

Luig, P.; Manchado-Lopez, C.; Perse, M.; Kristan, M.; Schander, I.; Zimmermann, M., et al. (2008). Motion characteristics according to playing position in international men's team handball. 13th Annual Congress of the European College of Sports Science, 255. Portugal: Estoril.

Madrera, E.; Herrero, J. A.; Fernández, A., y Martínez, I. (2003). Análisis videográfico de acciones específicas de fuerza en jugadores de campo de un equipo de balonmano. RendimientoDeportivo.com [En Línea], 4. Retrieved from http://www.rendimientodeportivo.com [Consulta 11/01/2007].

Manchado, C.; Hoffmann, E.; Navarro, F., y Platen, P. (2007). Beanspruchungsprofil im frauenhandball-belastungsdauer und herzfrequenzverhalten bei spielen der nationalmannschaft. Deutsche Zeitschrift Für Sportmedizin, 58(10), 368-373.

Marczinka, Z. (1993). Playing handball. Budapest: Trio Budapest.

Marques, M. C.; Van den Tillaar, R.; Vescovi, J. D., y González-Badillo, J. J. (2007). Relationship between throwing velocity, muscle power, and bar velocity during bench press in elite handball players. International Journal of Sports Physiology \& Performance, 2(4), 414-422.

Martínez, E. J. (2002). Pruebas de aptitud física. Barcelona: Paidotribo.

Mayo, C., y Pardo, A. (2001) Mejora de la fuerza específica a través del método de contraste: efectos sobre el rendimiento en jugadoras de balonmano de élite. II Congreso de Ciencias de la Actividad Física y el Deporte. Valencia: Facultad de Ciencias de la Actividad Física y el Deporte.

Mikkelsen, F., y Olensen, M. (1985). Etude physiologique du handball. Trygg-Hnasa Forbgverksamheten, 10626. Estocolmo

Moreno, F. (2004). Balonmano. detección, selección y rendimiento de talentos. Madrid: Gymnos.

Newton, R.U., y McEvoy, K.P. (1994) Baseball throwing velocity: a comparison of medicine ball training and weight training. Journal of Strength \& Conditioning Research, 8(3), 198-203.

Oxyzoglou, N.; Hatzimanouil, D.; Kanioglou, A., y Papadopoulou, Z. (2008). Profile of elite handball athletes by playing position. Physical Training, 24, 1-1.

Pardo, A.; González, L. M., y Mayo, C. (2007). Estudio de la cadena cinética del lanzamiento en salto en balonmano femenino ante situaciones de colaboración entre las defensoras y la portera. Selección: Revista Española De Medicina De La Educación Física y El Deporte, 16(2), 71-77.

Párraga, J.; Sánchez, A., y Oña, A. (2001). Importancia de la velocidad de salida del bálon y de la precisión como parámetros de eficacia en el lanzamiento en salto a distancia en balonmano. Apunts: Educación Física y Deportes, 66, 44-51.

Pezarat-Correia, P. L.; Valamatos, M. J.; Alves, F., y Santos, P. M. (2007). Influence of position roles on upper limb force parameters in young male handball players. Medicine \& Science in Sports \& Exercise, 39(5), 216-219.

Prokajac, B. (1980). Difference between initial ball velocities when using a sidearm throw in fieldball. Revista Física Cultura, 34, 333-337. 
Rivilla, J.; Martínez, M.; Navarro, F.; Sampedro, J. (2011). Diferencias en la distancia de lanzamiento y velocidad de balón según el puesto específico en jugadores de balonmano sub-18. Revista Internacional de Ciencias del Deporte, 21(6), 14-23. http://www.cafyd.com/REVISTA/02202.pdf

Rivilla, J.; Sampedro, J.; Navarro, F., y Gómez, M. J. (2010). Influencia de la oposición en la velocidad de lanzamiento en jugadores de balonmano de élite, amateur y formación. RICYDE. Revista Internacional De Ciencias Del Deporte, 6(18), 91-99.

Rivilla-García, J.; Díaz, A., y Sampedro, J. (2010). Estudio comparativo del uso de las Tic's en la enseñanza del balonmano en jugadores en formación. IX Congreso Internacional de Educación Física e Interculturalidad. Murcia: Facultad de Ciencias del Deporte.

Rogulj, N.; Srhoj, V.; Nazor, M.; Srhoj, L., y Cavala, M. (2005). Some anthropologic characteristics of elite female handball players at different playing positions. Collegium Antropologicum, 29(2), 705-709.

Ruiz, L.; Rodríguez, E., y Enrique, J. (2001). Estudio del somatotipo en jugadoras de balonmano por puestos y categorías. Apunts: Medicina De l'Esport, 36(137), 25-32.

Šibila, M.; Pori, P.; y Bon, M. (2003). Basic kinematic differences between two types of jump shot techniques in handball. Universitatis Palackianae Olomucensis Gymnica, $33(1), 19-26$.

Šibila, M.; Vuleta, D., y Pori, P. (2004). Position-related differences in volume and intensity of large-scale cyclic movements of male players in handball. Kinesiology, 36(1), 58-68.

Srhoj, V.; Marinovic, M., y Rogulj, N. (2002). Position specific morphological characteristics of top-level male handball players. Collegium Antropologicum, 26(1), 219-228.

Taborsky, F. (2007). Playing performance in team handball. Research Yearbook, 13(1), 156-159.

Torrescusa, L. C. (1986). Estudio sobre pruebas realizadas a jugadores de balonmano. Seminario De Balonmano Dirigido Por El Profesor Román Seco, Madrid: INEF-Madrid.

Van den Tillaar, R., y Ettema, G. (2003). Influence of instruction on velocity and accuracy of overarm throwing. Perceptual \& Motor Skills, 96(2), 423-434.

Van der Wende, K. (2005). The effects of game specific task constraints on the outcome of the water polo shot. (Tesis Doctoral, Auckland University of Technology). New Zealand: Faculty of Health and Environmental Science.

Van Muijen, A. E.; Joris, H.; Kemper, H. C. G., y Van Ingen Schenau, G. J. (1991). Throwing practice with different ball weights: Effects on throwing velocity and muscle strength in female handball players. Sports Training, Medicine \& Rehabilitation, 2(2), 103-113.

Vasques, D. G.; Antunes, P. C.; Silva, T. J., y Lopes, A. S. (2005). Morfologia de atletas de handebol: Comparação por posição ofensiva e defensiva de jogo. Lectura: Educación Física y Deportes [En Línea], 10(81). Retrieved from http://www.efdeportes.com [Consulta 19/07/2009].

Vila, H.; Fernández, J. J., y Rodríguez, F. A. (2007). Evolución de la condición física en jugadoras de balonmano en las categorías infantil, cadete y juvenil. Apunts: Educación Física y Deportes, 87, 99-106.

Vila, H.; Ferragut, C., y Alcaraz, P. E. (2008). Características cineantropométricas y la fuerza en jugadores juveniles de balonmano por puestos específicos. Archivos De Medicina Del Deporte, 25(125), 167-178.

Vila, H.; Ferragut, C.; Argudo, F. M.; Abraldes, J. A.; Rodríguez, N., y Alacid, F. (2009). Relación entre parámetros antropométricos y la velocidad de lanzamiento en jugadores de waterpolo. Journal of Human Sport and Exercise [En Línea], 4(1), 6274. Retrieved from http://www.jhse.com [Consulta 03/01/2010]. 
Rivilla, J.; Martínez, M.; Navarro, F.; Sampedro, J. (2011). Diferencias en la distancia de lanzamiento y velocidad de balón según el puesto específico en jugadores de balonmano sub-18. Revista Internacional de Ciencias del Deporte, 21(6), 14-23. http://www.cafyd.com/REVISTA/02202.pdf

Visnapuu, M., y Jurimae, T. (2008). The influence of basic body and hand anthropometry on the results of different throwing tests in young handball and basketball players. Anthropologischer Anzeiger; Bericht Uber Die Biologisch-Anthropologische Literatur, 66(2), 225-236.

Zapartidis, I.; Toganidis, T.; Vareltzis, I.; Christodoulidis, T.; Kororos, P., y Skoufas, D. (2009). Profile of young female handball players by playing position. Serbian Journal of Sports Sciences, 3(2), 53-60.

Zeier, U. (1987). As exigencies mínimas para a técnica do guarda-redes. Setemetros, 24, 29-33.

Ziv, G., y Lidor, R. (2009). Physical characteristics, physiological attributes, and on-court performances of handball players: A review. European Journal of Sport Science, 9(6), 375-386. 